\title{
Reactions of aldehydes with organozinc reagents derived from (E)-3-bromo- and (E)-3-iodo-1-phenylsulfonylprop-1-ene
}

\author{
Eva T. Gallagher and David H. Grayson*
}

School of Chemistry, Trinity Biomedical Sciences Institute, Trinity College Dublin 152-160

Pearse Street, Dublin 2, Ireland

E-mail: dgrayson@,tcd.ie

DOI: $\underline{\text { http://dx.doi.org/10.3998/ark.5550190.p008.741 }}$

\begin{abstract}
(E)-3-Bromo-1-phenylsulfonylprop-1-ene and (E)-3-iodo-1-phenylsulfonylprop-1-ene both form allylic organozinc reagents that react with aromatic aldehydes to give mainly 4-aryl-4-hydroxy1-phenylsulfonylbut-1-enes. In contrast, reactions of the same organozinc species with 2-methylpropanal or 3-methylbutanal gave mixtures of diastereoisomeric 4-hydroxy-1-phenylsulfonylalk1-enes and 4-hydroxy-3-phenylsulfonylalk-1-enes.
\end{abstract}

Keywords: Halo-sulfone, organozinc, aldehyde, alkylation

\section{Introduction}

We have previously reported ${ }^{1}$ that the (E)-3-halo-1-phenylsulfonylpropenes $\mathbf{1 - 3}$ and the (Z)-1halo-3-phenylsulfonylpropenes $\mathbf{4}$ and $\mathbf{5}$ can be lithiated to give hetero-substituted carbanions. These react with aldehydes to afford products arising from alkylation processes that occur either $\alpha$ - or $\gamma$ with respect to the phenylsulfonyl group.

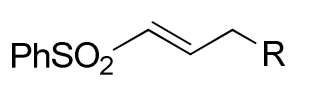

$$
\begin{aligned}
& 1 \mathrm{R}=\mathrm{Cl} \\
& 2 \mathrm{R}=\mathrm{Br} \\
& 3 \mathrm{R}=\mathrm{I}
\end{aligned}
$$<smiles>[R]/C=C\COS(=O)(=O)O</smiles>

$4 \mathrm{R}=\mathrm{Cl}$

$5 \mathrm{R}=\mathrm{Br}$

The structures of these products are dependent on the natures of both the halosulfone and the aldehyde. Thus, each of the chloro-compounds $\mathbf{1}$ and $\mathbf{4}$ form a common delocalised carbanion that reacts $\gamma$-selectively with aromatic aldehydes to give the hydroxysulfones $\mathbf{6}$. On the other 
hand, carbanions derived from the bromo- and iodosulfones $\mathbf{2}, \mathbf{5}$, and $\mathbf{3}$ react with aromatic aldehydes to yield epoxides 7. By contrast, when aliphatic aldehydes are used as electrophiles $\alpha$ alkylation of the chlorosulfone $\mathbf{4}$ takes place leading to formation of the anti-diastereoisomers of $\beta$-hydroxysulfones 8 .<smiles>O=S(=O)(C/C=C(\Cl)C(O)Br)c1ccccc1</smiles>

6<smiles>[Al]C1OC1C=CSOc1ccccc1</smiles>

7<smiles>[R]C(O)C(/C=C\Cl)[R6](=O)O</smiles>

8

The rich chemistry of the carbanions derivable from the halo-sulfone 1-3 suggested to us that other organometallic derivatives of these compounds might repay investigation. We now demonstrate that useful organozinc reagents can be prepared from the halosulfones $\mathbf{2}$ and $\mathbf{3}$, and that these react with aldehydes to yield Reformatsky-like products.

By analogy, 3-bromoprop-2-enoates have been reported ${ }^{2,3}$ to undergo Reformatsky reactions with aldehydes and with ketones to yield, depending upon the circumstances, either or both of the "normal" $\gamma$-alkylated products 9 and "abnormal" $\alpha$-alkylated products $\mathbf{1 0}$.<smiles>[R]OC(=O)C=CCC([R])O</smiles>

9<smiles>[R]OC(=O)C(C=C)C([R])O</smiles>

10

Steric effects are influential, ${ }^{3}$ and the $\gamma$-products are often produced under thermodynamic, and the $\alpha$-products under kinetic control. ${ }^{4-6}$

\section{Results and Discussion}

The allylic bromide 2 was treated in THF with zinc dust that had been activated either by treatment with hydrochloric acid followed by successive washings with organic solvents ${ }^{7}$ or by treatment with 1,2-dibromoethane followed by chlorotrimethylsilane. ${ }^{8}$ Subsequent reaction of the derived organozinc reagent with benzaldehyde yielded a mixture of the $(E)$ - $\gamma$-alkylated compound 11 together with a little of the anti-configured $\alpha$-alkylated product 12. The $\delta$-hydroxy vinylic sulfone $11, \mathrm{mp} 77^{\circ} \mathrm{C}$, had all of the spectroscopic features expected of it, and its $(E)$ configuration could be confidently assigned on the basis of the $J_{\text {trans }} 15.2 \mathrm{~Hz}$ coupling constant for its olefinic protons. The $\beta$-hydroxy sulfone $\mathbf{1 2}$ could not be isolated in pure form, but was readily identified from ${ }^{1} \mathrm{H}$ NMR data. In particular, as previously noted by us, ${ }^{1}$ the absence of 
any vicinal coupling between its two methine protons supports the anti-stereochemistry that is assigned to it.

The organozinc reagent derived from the bromosulfone 2 also reacted (Scheme 1) with 4-chlorobenzaldehyde to give a mixture of the $(E)$ - and $(Z)-\gamma$-alkylated products 13 and 14 in 93:7 ratio, with 3-fluorobenzaldehyde to give (by NMR) a mixture of $(E)-\mathbf{1 5},(Z)-16$ (minor) and anti-17, and with 3-methoxybenzaldehyde to give $(E)-\mathbf{1 8}$ and $(Z)-\mathbf{1 9}$. No reaction took place when either of 2-methoxybenzaldehyde or cyclohexanone was the electrophile, suggesting that the bromo-sulfone $\mathbf{2}$ does not form a particularly reactive organozinc species.

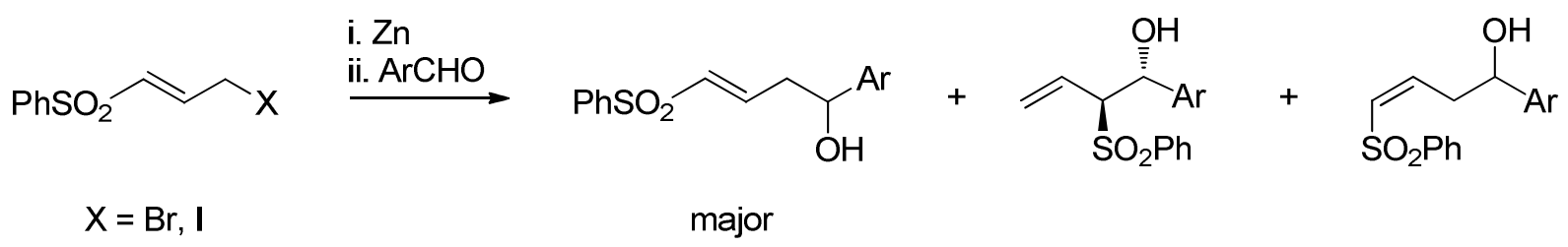<smiles>O=C(O)OC=CCC(O)[Al]</smiles><smiles>C=CC(O)C(O)C(Br)c1ccccc1</smiles><smiles>O=S(=O)(/C=C\CC(O)[Al])c1ccccc1</smiles>

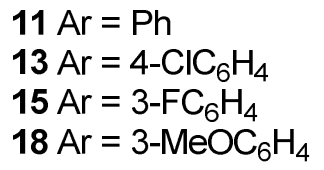

Scheme 1. Reactions of aryl aldehydes with the organozinc reagent derived from the halosulfones $\mathbf{2}$ and $\mathbf{3}$.

We also carried out similar reactions using the iodosulfone $\mathbf{3}$, with benzaldehyde and 4-chlorobenzaldehyde as the electrophiles, but yields were much poorer than when the bromosulfone 2 was employed.

By contrast (Scheme 2), the dominant "normal" regioselectivities observed for each of the above reactions were not seen when aliphatic aldehydes were the electrophiles. In addition, both diastereoisomers of each product bearing two asymmetric centres were produced, in contrast to the outcome when an aryl aldehyde was the electrophile. Thus, isobutyraldehyde yielded the four products 20-23 together with a little of the $(Z)$-bromide 24 when it was reacted with $(E)$-3bromo-1-phenylsulfonylprop-1-ene 2 in the presence of activated zinc. Each of the hydroxysulfones 20-23 was purified by column chromatography. The $(E)$ - and $(Z)$ - $\gamma$-alkylated compounds 20 and 21 were easily identified from their NMR spectra, as were the diastereoisomeric anti and syn $\alpha$-alkylated compounds 22 and 23 which have been previously reported by us. ${ }^{1}$ Similar results were obtained using isovaleraldehyde as the electrophile when the homologous $\gamma$-alkylated $\mathbf{2 5}$ and $\mathbf{2 6}$ and $\alpha$-alkylated $\mathbf{2 7}$ and $\mathbf{2 8}$ products were formed. 


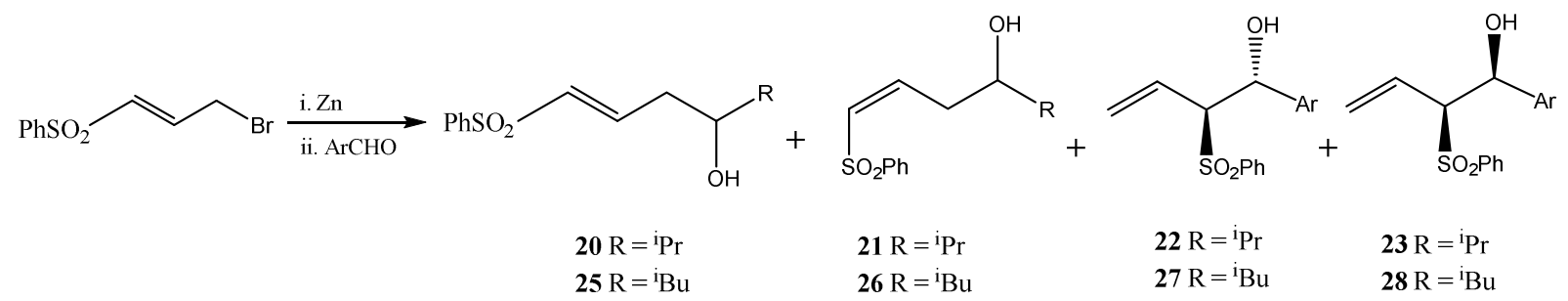

Scheme 2. Reactions of aliphatic aldehydes with the organozinc reagent derived from bromosulfone 2 .

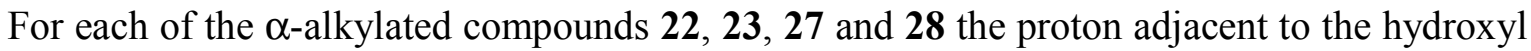
group resonates at lower field than expected (the range spans $\delta 4.1-4.6 \mathrm{ppm}$ ). We have previously observed ${ }^{1}$ that this may reflect strong conformational preferences in solution ( $c f$. our X-ray crystallographic analysis ${ }^{1}$ of a related compound, which showed no evidence for intramolecular hydrogen-bonding between the hydroxyl and sulfonyl groups in the solid state). We now note that for each of the anti-compounds $\mathbf{2 2}$ and $\mathbf{2 7}$ there is no vicinal coupling between H-3 and H-4, whereas for the syn-products $\mathbf{2 3}$ and $\mathbf{2 8}$ large vicinal coupling constants $J_{3,4}$ can be measured.

The results that we have obtained are summarised in Table 1, below, in which the data clearly illustrates the regioselectivity of reaction when aryl aldehydes are used as electrophiles.

Table 1. Reactions of aldehydes with the organozinc reagents derived from halosulfones $\mathbf{2}$ and 3

\begin{tabular}{|c|c|c|}
\hline Halosulfone & Aldehyde & Products \\
\hline 2 & benzaldehyde & $\mathbf{1 1}, 12(83: 17)$ \\
\hline 3 & benzaldehyde & $11^{a}$ \\
\hline 2 & 4-chlorobenzaldehyde & 13, $14(93: 7)$ \\
\hline 3 & 4-chlorobenzaldehyde & $13^{a}$ \\
\hline 2 & 3-fluorobenzaldehyde & $\mathbf{1 5}, 17(90: 10)$ \\
\hline 2 & 3-methoxybenzaldehyde & 18, $19(91: 9)$ \\
\hline 2 & isobutyraldehyde & $20,21,22,23(46: 14: 35: 5)$ \\
\hline 2 & isovaleraldehyde & $\mathbf{2 5}, \mathbf{2 6}, \mathbf{2 7}, \mathbf{2 8}(29: 15: 33: 23)$ \\
\hline
\end{tabular}

${ }^{a}$ The product was obtained in poor yield.

3-Phenylsulfonylprop-1-ene $\mathbf{2 9}$ was an occasional side product in some reactions of the organozinc reagent derived from the bromide 2. In a control experiment, this was quenched with water to give the $\alpha$-protonated compound 1-phenylsulfonylprop-2-ene 29 as the only product. 
<smiles>O=[R6](O)OC/C=C\Br</smiles>

24<smiles>C=CCOS(=O)(=O)O</smiles>

29

Taken together with the results described above, we conclude that steric factors are dominant in controlling the regioselectivity of the reaction of this reagent with electrophiles.

\section{Conclusions}

Organozinc reagents prepared from (E)-3-bromo-1-phenylsulfonylprop-1-ene 2 and from $(E)$-3iodo-1-phenylsulfonylprop-1-ene $\mathbf{3}$ react with aryl aldehydes with good specificity to yield useful phenylsulfonyl-substituted homoallylic alcohols. However, similar reactions with representative aliphatic aldehydes lead to mixtures of regio- and diastereoisomeric products. The bromosulfone $\mathbf{2}$ gives superior results to the iodosulfone $\mathbf{3}$ in all of these reactions.

\section{Experimental Section}

General. Unless otherwise stated, ${ }^{1} \mathrm{H}$ NMR spectra were recorded for solutions in $\mathrm{CDCl}_{3}$ using JEOL PMX-60, Bruker WP-80, Bruker MSL $300 \mathrm{MHz}$ or Bruker Avance DPX $400 \mathrm{MHz}$ spectrometers. Coupling constants are recorded in Hz. Assignments were verified where appropriate by ${ }^{1} \mathrm{H}-{ }^{1} \mathrm{H}$ COSY, ${ }^{1} \mathrm{H}_{-}{ }^{13} \mathrm{C}$ COSY and DEPT experiments. IR spectra were recorded for Nujol mulls $(\mathrm{N})$ or liquid films (L) between sodium chloride plates using Perkin Elmer 883 or Paragon 1000 spectrometers. Mass spectra were obtained Kratos or Micromass instruments. Melting points (uncorrected) were measured in unsealed capillary tubes using a Stuart Scientific SMP2 digital apparatus or an Electrothermal IA9100 apparatus. Thin layer chromatography was carried out using Merck Kieselgel $60 \mathrm{~F}_{254} 0.2 \mathrm{~mm}$ silica gel plates. Column chromatography was carried out using Merck Kieselgel 60 (70-230 mesh) silica gel. All solvents were dried and distilled before use. Ethereal extracts of reaction products were dried over anhydrous magnesium sulfate. Combustion analyses were obtained from the Microanalytical Laboratory, University College, Dublin.

(E)-3-Bromo-1-phenylsulfonylprop-1-ene (2). This was prepared as previously described ${ }^{1,10}$ and had $\mathrm{mp} 50-51{ }^{\circ} \mathrm{C}$ (lit., ${ }^{9} \mathrm{mp} 34-35{ }^{\circ} \mathrm{C}$ ).

(E)-3-Iodo-1-phenylsulfonylprop-1-ene (3). This was prepared as previously described ${ }^{1,10}$ and had mp 62-63 ${ }^{\circ} \mathrm{C}$ (lit., ${ }^{9} \mathrm{mp} 67-68^{\circ} \mathrm{C}$ ). 


\section{General procedures for generation of organozinc reagents and their reaction with aldehydes}

Procedure A. Zinc dust was treated ${ }^{7}$ with dilute hydrochloric acid and then washed sequentially with water, ethanol, acetone and ether before being dried in a vacuum desiccator. Some of a solution of either of the halosulfones 2 or $3(2 \mathrm{mmol})$ and an aldehyde $(2 \mathrm{mmol})$ in benzene (10 $\mathrm{mL}$ ) was added dropwise, under dry nitrogen, to the purified zinc (10 mmol, 5 equiv.), and the mixture was warmed until reaction was apparent. The remainder of the solution of reactants was then introduced at such a rate that gentle reflux was maintained. After $0.5 \mathrm{~h}$ at reflux, the mixture was poured into ice-cold sulfuric acid (10\%). The organic phase was separated, and the aqueous phase was extracted with ether. The combined organic phases were then dried and evaporated.

Procedure B. Zinc dust ( 0.6 g, 5 equiv.) was stirred ${ }^{8}$ under nitrogen with dibromoethane (70 $\mathrm{mg})$ in dry THF $(2 \mathrm{~mL})$ and the suspension was put through a heating-stirring $(1 \mathrm{~min})$-cooling cycle in triplicate. Chlorotrimethylsilane $(70 \mu \mathrm{L})$ was then added followed, after a few minutes by a solution of one of the halosulfones 2 or $3(2 \mathrm{mmol})$ and an aldehyde $(2 \mathrm{mmol})$ in dry THF (5 $\mathrm{mL}$ ). After $2 \mathrm{~h}$ at reflux, the mixture was cooled, diluted with ether and poured into ice-cold sulfuric acid (10\%). The organic phase was separated, and the aqueous phase was extracted with ether. The combined organic phases were then dried and evaporated.

(E)-4-Hydroxy-4-phenyl-1-phenylsulfonylbut-1-ene (11) and 3,4-anti-4-hydroxy-4-phenyl3-phenylsulfonylbut-1-ene (12). Using procedure B, the bromosulfone $2(0.52 \mathrm{~g})$ and benzaldehyde $(0.21 \mathrm{~g})$ reacted to give product $(0.48 \mathrm{~g})$ that $\left({ }^{1} \mathrm{H} \mathrm{NMR}\right)$ contained the hydroxysulfones $11(83 \%)$ and $\mathbf{1 2}(17 \%)$.

(E)-4-Hydroxy-4-phenyl-1-phenylsulfonylbut-1-ene (11). This was obtained by fractional crystallisation of the crude product mixture and had $\mathrm{mp} 77^{\circ} \mathrm{C}\left(\mathrm{Et}_{2} \mathrm{O}\right), v_{\max }(\mathrm{N}) 3512,1306$ and $1144 \mathrm{~cm}^{-1} ; \delta_{\mathrm{H}}(300 \mathrm{MHz}) 2.62\left(1 \mathrm{H}, \mathrm{s}\right.$, exch. $\left.\mathrm{D}_{2} \mathrm{O},-\mathrm{OH}\right), 2.63\left(2 \mathrm{H}, \mathrm{m},-\mathrm{CH}_{2} \mathrm{CH}=\mathrm{CH}\right), 4.82(1 \mathrm{H}, \mathrm{t}$, $J$ 6.3, $-\mathrm{CHOH}), 6.32\left(1 \mathrm{H}, \mathrm{d}, J 15.1,-\mathrm{SO}_{2} \mathrm{CH}=\mathrm{CH}\right), 6.99(1 \mathrm{H}, \mathrm{dt}, J 15.1,7.3$ and 7.3, $\left.\mathrm{SO}_{2} \mathrm{CH}=\mathrm{CHCH}_{2}\right), 7.27(5 \mathrm{H}, \mathrm{m}, \mathrm{Ar} H), 7.61\left(1 \mathrm{H}, \mathrm{t}, J 7.5, p-\left(\mathrm{SO}_{2}\right) \mathrm{Ar} H\right)$ and $7.79(2 \mathrm{H}, \mathrm{d}, J$ 8.0, $o-$ $\left.\left(\mathrm{SO}_{2}\right) \mathrm{ArH}\right) \mathrm{ppm} ; \delta_{\mathrm{C}}(75 \mathrm{MHz}) 40.7\left(\mathrm{CH}_{2}\right), 72.5(\mathrm{CHOH}), 125.6(\mathrm{CH}), 127.5(\mathrm{CH}), 127.8(\mathrm{CH})$, $128.9(\mathrm{CH}), 129.2(\mathrm{CH}), 132.6(\mathrm{CH}), 133.2(\mathrm{CH}=\mathrm{C}), 134.0\left(C_{\mathrm{q}}\right), 140.3\left(C_{\mathrm{q}}\right)$ and $142.9(-$ $\mathrm{SO}_{2} \mathrm{CH}=C H$ ) ppm. Anal. Calc. for $\mathrm{C}_{16} \mathrm{H}_{16} \mathrm{O}_{3} \mathrm{~S}: \mathrm{C} 66.64, \mathrm{H} 5.59 \%$. Found: C 66.43, H 5.57\%.

The minor product, 3,4-anti-4-hydroxy-4-phenyl-3-phenylsulfonylbut-1-ene (12), has been previously described by us ${ }^{1}$ and was identified from its ${ }^{1} \mathrm{H}$ NMR spectrum and by tlc comparison with authentic material.

Poorer results were obtained by using procedure A, when the hydroxysulfone $\mathbf{1 1}$ was isolated in $19 \%$ yield from the bromosulfone 2 or in $20 \%$ yield from the iodosulfone 3 .

(E)-4-(4'-Chlorophenyl)-4-hydroxy-1-phenylsulfonylbut-1-ene (13). Using procedure B, the bromosulfone $2(0.52 \mathrm{~g})$ and 4-chlorobenzaldehyde $(0.29 \mathrm{~g})$ reacted to give a crude product $(0.51$ g) that was chromatographed using ether-hexane as eluant to give an oil $(0.38 \mathrm{~g}, 59 \%)$ that $\left({ }^{1} \mathrm{H}\right.$ NMR) contained (E)-4-(4'-chlorophenyl)-4-hydroxy-1-phenylsulfonylbut-1-ene (13) (93\%) and its $(Z)$-isomer 14 (7\%) from which 13 could not be further separated. 
(E)-4-(4'-Chlorophenyl)-4-hydroxy-1-phenylsulfonylbut-1-ene (13) had $\boldsymbol{v}_{\max }$ (N) 3510, 1307 and $1143 \mathrm{~cm}^{-1} ; \delta_{\mathrm{H}}(300 \mathrm{MHz}) 2.60\left(2 \mathrm{H}, \mathrm{m},-\mathrm{CH}_{2} \mathrm{CH}=\mathrm{CH}\right), 2.90\left(1 \mathrm{H}, \mathrm{s}\right.$, exch. $\left.\mathrm{D}_{2} \mathrm{O},-\mathrm{OH}\right), 4.81$ $(1 \mathrm{H}, \mathrm{dd}, J 7.1$ and 5.5, $-\mathrm{CHOH}), 6.36\left(1 \mathrm{H}, \mathrm{d}, J 15.0,-\mathrm{SO}_{2} \mathrm{CH}=\mathrm{CH}\right), 6.93(1 \mathrm{H}, \mathrm{dt}, J 15.0,7.3$ and 7.3, $\left.-\mathrm{SO}_{2} \mathrm{CH}=\mathrm{CHCH} \mathrm{CH}_{2}\right), 7.20(4 \mathrm{H}, \mathrm{m}, \mathrm{Cl}-\mathrm{Ar} H), 7.50\left(2 \mathrm{H}, \mathrm{m}, m-\left(\mathrm{SO}_{2}\right) \mathrm{Ar} H\right), 7.60(1 \mathrm{H}, \mathrm{t}, J 7.5, p-$ $\left.\left(\mathrm{SO}_{2}\right) \mathrm{ArH}\right)$ and $7.74\left(2 \mathrm{H}, \mathrm{d}, J 8.0, o-\left(\mathrm{SO}_{2}\right) \mathrm{ArH}\right) \mathrm{ppm} ; \delta_{\mathrm{C}}(75 \mathrm{MHz}) 40.7\left(\mathrm{CH}_{2}\right), 71.6(\mathrm{CHOH})$, 127.0 $(\mathrm{CH}), 127.1(\mathrm{CH}), 128.6(\mathrm{CH}), 129.2(\mathrm{CH}), 132.7(\mathrm{CH}), 133.2(\mathrm{CH}=\mathrm{C}), 133.5\left(C_{\mathrm{q}}\right), 141.4$ $\left(C_{\mathrm{q}}\right)$ and $142.6\left(-\mathrm{SO}_{2} \mathrm{CH}=C \mathrm{H}\right) \mathrm{ppm}$. Anal. Calc. for $\mathrm{C}_{16} \mathrm{H}_{15} \mathrm{ClO}_{3} \mathrm{~S}: \mathrm{C} 59.53, \mathrm{H} 4.65 \%$. Found (for the 93 : 7 mixture of diastereoisomers): $\mathrm{C} 59.52, \mathrm{H} 4.73 \%$.

Using procedure $\mathrm{A}$, the iodosulfone $\mathbf{3}$ and 4-chlorobenzaldehyde gave the hydroxysulfone $\mathbf{1 3}$ in $22 \%$ yield.

(E)-4-(3'-Fluorophenyl)-4-hydroxy-1-phenylsulfonylbut-1-ene (15). Using procedure B, the bromosulfone $2(0.52 \mathrm{~g})$ and 3-fluorobenzaldehyde $(0.26 \mathrm{~g})$ reacted to give product $(0.56 \mathrm{~g})$ that (NMR) contained the hydroxysulfone 15 together with ca. 10\% of 3,4-anti-4-(3'-fluorophenyl)4-hydroxy-3-phenylsulfonylbut-1-ene (17). The gross structure of 17 was clear from its spectrum, and its stereochemistry could be inferred from ${ }^{1}$ the near-zero value of $J_{3,4}$. The crude product was recrystallised from ether-hexane to give pure $(E)$-4-(3'-fluorophenyl)-4-hydroxy-1phenylsulfonylbut-1-ene (15), (0.37 g, 60\%), mp $197{ }^{\circ} \mathrm{C}, v_{\max }(\mathrm{N}) 3500,1305$ and $1149 \mathrm{~cm}^{-1} ; \delta_{\mathrm{H}}$ (300 MHz) $2.61\left(2 \mathrm{H}, \mathrm{m},-\mathrm{CH}_{2} \mathrm{CH}=\mathrm{CH}\right), 2.97\left(1 \mathrm{H}, \mathrm{s}\right.$, exch. $\left.\mathrm{D}_{2} \mathrm{O},-\mathrm{OH}\right), 4.83(1 \mathrm{H}$, br t, J 7.0, $\mathrm{CHOH}), 6.33\left(1 \mathrm{H}, \mathrm{dd}, J 15.0\right.$ and 1.3, $\left.-\mathrm{SO}_{2} \mathrm{CH}=\mathrm{CH}\right), 6.9-7.03(4 \mathrm{H}$, overlapping $\mathrm{ms}$, $\mathrm{SO}_{2} \mathrm{CH}=\mathrm{CHCH}_{2}, o-(\mathrm{F}) \mathrm{Ar} H$ and $\left.p-(\mathrm{F}) \mathrm{Ar} H\right), 7.24(1 \mathrm{H}, \mathrm{m}, \mathrm{m}-(\mathrm{F}) \mathrm{Ar} H), 7.50\left(2 \mathrm{H}, \mathrm{m}, \mathrm{m}-\left(\mathrm{SO}_{2}\right) \mathrm{Ar} H\right)$, $7.59\left(1 \mathrm{H}, \mathrm{t}, J 7.5, \mathrm{p}-\left(\mathrm{SO}_{2}\right) \mathrm{ArH}\right)$ and $7.77\left(2 \mathrm{H}, \mathrm{d}, J 8.0, \mathrm{o}-\left(\mathrm{SO}_{2}\right) \mathrm{Ar} H\right) \mathrm{ppm} ; \delta_{\mathrm{C}}(75 \mathrm{MHz}) 40.7$ $\left(\mathrm{CH}_{2}\right), 71.7(\mathrm{CHOH}), 112.4(\mathrm{~d}, J$ 22.0, $o-(\mathrm{F}) \mathrm{ArCH}), 114.6(\mathrm{~d}, J$ 21.0, $o-(\mathrm{F}) \mathrm{ArCH}), 121.2(p-$ (F)ArCH), $127.4(\mathrm{CH}), 129.2(\mathrm{CH}), 130.1(\mathrm{~d}, J$ 8.0, m-(F)ArCH), $132.6(\mathrm{CH}), 133.4(\mathrm{CH}=\mathrm{C})$, $139.9\left(C_{\mathrm{q}}\right), 142.5\left(-\mathrm{SO}_{2} \mathrm{CH}=\mathrm{CH}\right), 145.6\left(C_{\mathrm{q}}, \mathrm{d}, J 7.0\right)$ and $162.0\left(C_{\mathrm{q}}, \mathrm{d}, J 245\right)$ ppm. Anal. Calc. for $\mathrm{C}_{16} \mathrm{H}_{15} \mathrm{FO}_{3} \mathrm{~S}$ : C 62.75, $\mathrm{H} 4.90 \%$. Found: C 63.01, $\mathrm{H} 4.69 \%$.

(E)-4-(3'-Methoxyphenyl)-4-hydroxy-1-phenylsulfonylbut-1-ene (18). Using procedure B, the bromosulfone $2(0.52 \mathrm{~g})$ and 3-methoxybenzaldehyde $(0.26 \mathrm{~g})$ reacted to give an oily product $(0.57 \mathrm{~g})$ that $(\mathrm{NMR})$ contained the hydroxysulfone 18 together with $c a .9 \%$ of its $(Z)$-isomer $\mathbf{1 9}$. This mixture was chromatographed using ether-hexane as eluant to give $\mathbf{1 8}$ as an oil (0.36 g; 57\%) from which some remaining 19 could not be further separated. (E)-4-(3'-Methoxyphenyl)4-hydroxy-1-phenylsulfonylbut-1-ene (18) had $v_{\max }(\mathrm{N}) 3494,1306$ and $1147 \mathrm{~cm}^{-1} ; \delta_{\mathrm{H}}(300$ $\mathrm{MHz}) 2.61\left(2 \mathrm{H}, \mathrm{m},-\mathrm{CH}_{2} \mathrm{CH}=\mathrm{CH}\right), 2.75\left(1 \mathrm{H}, \mathrm{s}\right.$, exch. $\left.\mathrm{D}_{2} \mathrm{O},-\mathrm{OH}\right), 3.77\left(3 \mathrm{H}, \mathrm{s},-\mathrm{OCH}_{3}\right), 4.79(1 \mathrm{H}$, t, $J$ 7.1, $-\mathrm{CHOH}), 6.32\left(1 \mathrm{H}, \mathrm{dd}, J 15.1\right.$ and 1.0, $\left.-\mathrm{SO}_{2} \mathrm{CH}=\mathrm{CH}\right), 6.89(2 \mathrm{H}, \mathrm{m},(\mathrm{MeO}) \mathrm{ArH}), 6.94$ $(1 \mathrm{H}, \mathrm{m},(\mathrm{MeO}) \mathrm{ArH}), 6.99\left(1 \mathrm{H}, \mathrm{dt}, J 15.1,7.3\right.$ and $\left.7.3,-\mathrm{SO}_{2} \mathrm{CH}=\mathrm{CHCH}_{2}\right), 7.25(1 \mathrm{H}, \mathrm{m}, m-$ (MeO)ArH), $7.52\left(2 \mathrm{H}, \mathrm{m}, m-\left(\mathrm{SO}_{2}\right) \mathrm{ArH}\right), 7.59\left(1 \mathrm{H}, \mathrm{t}, J\right.$ 7.5, $p$ - $\left.\left(\mathrm{SO}_{2}\right) \mathrm{Ar} H\right)$ and $7.82(2 \mathrm{H}, \mathrm{d}, J$ 8.0, $\left.o-\left(\mathrm{SO}_{2}\right) \mathrm{Ar} H\right) \mathrm{ppm} ; \delta_{\mathrm{C}}(75 \mathrm{MHz}) 40.7\left(\mathrm{CH}_{2}\right), 55.14\left(\mathrm{CH}_{3}\right), 72.3(\mathrm{CHOH}), 111.0(\mathrm{CH}), 113.2$ $(\mathrm{CH}), 117.9(\mathrm{CH}), 127.4(\mathrm{CH}), 127.4(\mathrm{CH}), 129.2(\mathrm{CH}), 130.1$ (d, J 8.0, m-(F)ArCH), 129.2 $(C H), 129.6(C H), 133.2(\mathrm{CH}=\mathrm{C}), 140.3\left(C_{\mathrm{q}}\right), 143.0\left(\mathrm{SO}_{2} \mathrm{CH}=C \mathrm{H}\right), 144.7\left(C_{\mathrm{q}}\right)$ and $159.7\left(C_{\mathrm{q}}\right)$ ppm. HRMS m/z 318.0925. Calc. for $\mathrm{C}_{17} \mathrm{H}_{18} \mathrm{O}_{4} \mathrm{~S}: 318.0926$. 
Reaction of isobutyraldehyde with the organozinc reagent derived from (E)-1-phenylsulfonyl-3-bromoprop-1-ene 2. Using procedure B, the bromosulfone $2(0.52 \mathrm{~g})$ and isobutyraldehyde $(0.14 \mathrm{~g})$ reacted to give an oily product mixture $(0.51 \mathrm{~g})$ that contained (NMR) 3-phenylsulfonylprop-1-ene 29 (13\%), the sulfone $\mathbf{2 4}^{1}(6.5 \%)$ and all four of the possible Reformatsky products 20 (37\%), 21 (11\%), 22 (28\%) and 23 (4\%). This mixture was chromatographed (EtOAc/n-hexane) to afford pure samples of each of the latter products.

(E)-4-Hydroxy-5-methyl-1-phenylsulfonylhex-1-ene (20). Obtained as an oil, $v_{\max }$ (L) 3521, 1306 and $1148 \mathrm{~cm}^{-1} ; \delta_{\mathrm{H}}(400 \mathrm{MHz}) 0.88\left(6 \mathrm{H}, \mathrm{d}, J 6.8,-\mathrm{CH}_{3}\right), 1.64\left(1 \mathrm{H}, \mathrm{m},-\mathrm{CH}\left(\mathrm{CH}_{3}\right)_{2}\right), 2.37$ $\left(2 \mathrm{H}, \mathrm{m},-\mathrm{CH}_{2} \mathrm{CH}=\mathrm{CH}\right), 2.50\left(1 \mathrm{H}, \mathrm{s}\right.$, exch. $\left.\mathrm{D}_{2} \mathrm{O},-\mathrm{OH}\right), 3.50(1 \mathrm{H}, \mathrm{m},-\mathrm{CHOH}-), 6.43(1 \mathrm{H}, \mathrm{dd}, J$ 15.1 and 1.2, $\left.-\mathrm{SO}_{2} \mathrm{CH}=\mathrm{CH}\right), 7.06\left(1 \mathrm{H}, \mathrm{dt}, J 15.1,7.7\right.$ and $\left.7.7,-\mathrm{SO}_{2} \mathrm{CH}=\mathrm{CHCH}_{2}-\right), 7.5(2 \mathrm{H}, \mathrm{m}$, $\operatorname{ArH}), 7.59(1 \mathrm{H}, \mathrm{m}, \mathrm{Ar} H)$ and $7.86\left(2 \mathrm{H}, \mathrm{d}, J\right.$ 8.3,o-ArH) ppm; $\delta_{\mathrm{C}}(100 \mathrm{MHz}) 16.9\left(\mathrm{CH}_{3}\right), 18.1$ $\left(\mathrm{CH}_{3}\right), 33.2\left(-\mathrm{CHMe}_{2}\right), 35.8\left(\mathrm{CH}_{2}\right), 74.4(-\mathrm{CHOH}-), 127.9(\mathrm{CH}), 128.8(\mathrm{CH}), 131.4(-$ $\left.\mathrm{SO}_{2} \mathrm{CH}=\mathrm{CH}\right), 132.9(\mathrm{CH}), 139.9\left(C_{\mathrm{q}}\right)$ and $144.4\left(\mathrm{SO}_{2} \mathrm{CH}=\mathrm{CH}\right)$ ppm. Anal. Calc. for $\mathrm{C}_{13} \mathrm{H}_{18} \mathrm{O}_{3} \mathrm{~S}$ : C 61.42, H 7.09\%. Found: C 61.22, H 7.14\%.

(Z)-4-Hydroxy-5-methyl-1-phenylsulfonylhex-1-ene (21). Obtained as an oil, $v_{\max }$ (L) 3500, 1305 and $1149 \mathrm{~cm}^{-1} ; \delta_{\mathrm{H}}(400 \mathrm{MHz}) 0.97\left(6 \mathrm{H}\right.$, apparent t, $J 5.9$ and 5.6, $\left.-\mathrm{CH}_{3}\right), 1.72(1 \mathrm{H}, \mathrm{m},-$ $\left.\mathrm{C} H \mathrm{Me}_{2}\right), 1.87\left(1 \mathrm{H}, \mathrm{s}\right.$, exch. $\left.\mathrm{D}_{2} \mathrm{O},-\mathrm{OH}\right), 2.85\left(2 \mathrm{H}, \mathrm{m},-\mathrm{CH}_{2} \mathrm{CH}=\mathrm{CH}\right), 3.49(1 \mathrm{H}, \mathrm{m},-\mathrm{CHOH}-)$, $6.45\left(2 \mathrm{H}\right.$, overlapping ms, $\left.-\mathrm{SO}_{2} \mathrm{CH}=\mathrm{CH}\right), 7.57(2 \mathrm{H}, \mathrm{m}, \mathrm{ArH}), 7.64(1 \mathrm{H}, \mathrm{m}, \mathrm{ArH})$ and $7.94(2 \mathrm{H}$, d, $J$ 8.3, o-ArH) ppm; $\delta_{\mathrm{C}}(100 \mathrm{MHz}) 17.0\left(\mathrm{CH}_{3}\right), 18.1\left(\mathrm{CH}_{3}\right), 32.0\left(-\mathrm{CHMe}_{2}\right), 33.6\left(\mathrm{CH}_{2}\right), 75.2(-$ $C \mathrm{HOH}-), 126.8(\mathrm{CH}), 128.8(\mathrm{CH}), 131.3\left(-\mathrm{SO}_{2} \mathrm{CH}=\mathrm{CH}\right), 133.0(\mathrm{CH}), 141.1\left(C_{\mathrm{q}}\right)$ and 143.8 $\left(\mathrm{SO}_{2} \mathrm{CH}=C \mathrm{H}\right) \mathrm{ppm}$. HRMS $m / z$ 254.0977. Calc. for $\mathrm{C}_{13} \mathrm{H}_{18} \mathrm{O}_{3} \mathrm{~S}: 254.0977$.

3,4-anti-4-Hydroxy-5-methyl-3-phenylsulfonylhex-1-ene (22). mp 88-89 ${ }^{\circ} \mathrm{C}$ ( $\mathrm{Et}_{2} \mathrm{O} / n$-pentane) was spectroscopically identical with authentic material previously characterized. ${ }^{1}$

3,4-syn-4-Hydroxy-5-methyl-3-phenylsulfonylhex-1-ene (23). colourless oil that was spectroscopically identical with authentic material previously characterized. ${ }^{1}$

Reaction between isovaleraldehyde and the organozinc reagent derived from $(E)$-1phenylsulfonyl-3-bromoprop-1-ene 2

Using procedure $\mathrm{B}$, the bromosulfone $2(0.52 \mathrm{~g})$ and isovaleraldehyde $(0.17 \mathrm{~g})$ reacted to give an oily product mixture $(0.51 \mathrm{~g})$ that contained (NMR) 3-phenylsulfonylprop-1-ene $29(9 \%)$, the sulfone 2 (3.5\%) and all four of the possible Reformatsky products $25(25 \%), 26(12.5 \%), 27$ (28\%) and 28 (20\%). This mixture was chromatographed (EtOAc/n-hexane) to afford pure samples of each of the latter products.

(E)-4-Hydroxy-6-methyl-1-phenylsulfonylhept-1-ene (25). Obtained as an oil, $v_{\max }$ (L) 3518, 1306 and $1146 \mathrm{~cm}^{-1} ; \delta_{\mathrm{H}}(400 \mathrm{MHz}) 0.89\left(3 \mathrm{H}, \mathrm{d}, J 6.6,-\mathrm{CH}_{3}\right), 0.90\left(3 \mathrm{H}, \mathrm{d}, J 6.6,-\mathrm{CH}_{3}\right), 1.23$ $\left(1 \mathrm{H}, \mathrm{m},-\mathrm{CHOH}-\mathrm{CH} H_{a}\right), 1.42\left(1 \mathrm{H}, \mathrm{m},-\mathrm{CHOH}-\mathrm{CH} H_{b}\right), 1.75(1 \mathrm{H}, \mathrm{m},-\mathrm{CHMe}), 1.91(1 \mathrm{H}, \mathrm{s}$, exch. $\left.\mathrm{D}_{2} \mathrm{O},-\mathrm{OH}\right), 2.37\left(2 \mathrm{H}, \mathrm{m},-\mathrm{CH}_{2} \mathrm{CH}=\mathrm{CH}\right), 3.86(1 \mathrm{H}, \mathrm{m},-\mathrm{CHOH}-), 6.44(1 \mathrm{H}, \mathrm{dd}, J 15.1$ and 1.5, $\left.\mathrm{SO}_{2} \mathrm{CH}=\mathrm{CH}\right), 7.05\left(1 \mathrm{H}, \mathrm{dt}, J 15.1,7.0\right.$ and $\left.7.0,-\mathrm{SO}_{2} \mathrm{CH}=\mathrm{CHCH}_{2}-\right), 7.54(2 \mathrm{H}, \mathrm{m}, \mathrm{ArH}), 7.62(1 \mathrm{H}$, $\mathrm{m}, \mathrm{Ar} H)$ and $7.90(2 \mathrm{H}, \mathrm{d}, J 8.0, o-\mathrm{Ar} H) \mathrm{ppm} ; \delta_{\mathrm{C}}(100 \mathrm{MHz}) 21.5\left(\mathrm{CH}_{3}\right), 22.8\left(\mathrm{CH}_{3}\right), 24.1(-$ $\left.\mathrm{CHMe}_{2}\right), 39.3\left(\mathrm{CH}_{2}\right), 45.9\left(\mathrm{CH}_{2}\right), 67.8(-\mathrm{CHOH}-), 127.1(\mathrm{CH}), 128.8(\mathrm{CH}), 132.0 \quad(-$ 
$\left.\mathrm{SO}_{2} \mathrm{CH}=\mathrm{CH}\right), 132.8(\mathrm{CH}), 140.1\left(C_{\mathrm{q}}\right)$ and $143.2\left(\mathrm{SO}_{2} \mathrm{CH}=C \mathrm{H}\right)$ ppm. HRMS $m / z$ 269.1209. Calc. for $\left[\mathrm{C}_{14} \mathrm{H}_{20} \mathrm{O}_{3} \mathrm{~S}+\mathrm{H}\right]^{+}: 269.1211$.

(Z)-4-Hydroxy-6-methyl-1-phenylsulfonylhept-1-ene (26). Obtained as an oil, $v_{\max }$ (L) 3518, 1306 and $1145 \mathrm{~cm}^{-1} ; \delta_{\mathrm{H}}(400 \mathrm{MHz}) 0.94\left(6 \mathrm{H}\right.$, apparent $\mathrm{t}, J 7.1$ and $\left.6.9,-\mathrm{CH}_{3}\right), 1.30(1 \mathrm{H}, \mathrm{m},-$ CHOH-CHH $\left.H_{a}\right), 1.45\left(1 \mathrm{H}, \mathrm{m},-\mathrm{CHOH}-\mathrm{CH} H_{b}\right), 1.77\left(2 \mathrm{H}, \mathrm{m},-\mathrm{CH} \mathrm{Me}_{2}\right.$ and [exch. $\mathrm{D}_{2} \mathrm{O}$ ] $\left.-\mathrm{OH}\right), 2.87$ $\left(2 \mathrm{H}, \mathrm{m},-\mathrm{CH}_{2} \mathrm{CH}=\mathrm{CH}\right), 3.86(1 \mathrm{H}, \mathrm{m},-\mathrm{CHOH}-), 6.39\left(2 \mathrm{H}, \mathrm{m}\right.$, contains d, $\left.J 13.6,-\mathrm{SO}_{2} \mathrm{CH}=\mathrm{CH}\right)$, $7.57(2 \mathrm{H}, \mathrm{m}, \mathrm{Ar} H), 7.65(1 \mathrm{H}, \mathrm{m}, \mathrm{Ar} H)$ and $7.95(2 \mathrm{H}, \mathrm{d}, J 8.3, o-\operatorname{Ar} H) \mathrm{ppm} ; \delta_{\mathrm{C}}(100 \mathrm{MHz}) 21.6$ $\left(\mathrm{CH}_{3}\right), 22.8\left(\mathrm{CH}_{3}\right), 24.1\left(-\mathrm{CHMe}_{2}\right), 35.2\left(\mathrm{CH}_{2}\right), 46.2\left(\mathrm{CH}_{2}\right), 68.4(-\mathrm{CHOH}-), 126.8(\mathrm{CH}), 128.8$ $(C H), 131.5\left(-\mathrm{SO}_{2} \mathrm{CH}=\mathrm{CH}\right), 133.0(\mathrm{CH}), 140.1\left(C_{\mathrm{q}}\right)$ and $143.0\left(\mathrm{SO}_{2} \mathrm{CH}=C \mathrm{H}\right) \mathrm{ppm}$. HRMS $\mathrm{m} / z$ 269.1218. Calc. for $\left[\mathrm{C}_{14} \mathrm{H}_{20} \mathrm{O}_{3} \mathrm{~S}+\mathrm{H}\right]^{+}: 269.1211$.

3,4-anti-4-Hydroxy-6-methyl-3-phenylsulfonylhept-1-ene (27). ${ }^{1}$ Obtained as an oil, $v_{\max }(\mathrm{L})$ 3518,1305 and $1149 \mathrm{~cm}^{-1} ; \delta_{\mathrm{H}}(400 \mathrm{MHz}) 0.89\left(3 \mathrm{H}, \mathrm{d}, J 6.5,-\mathrm{CH}_{3}\right), 0.91\left(3 \mathrm{H}, \mathrm{d}, J 7.0,-\mathrm{CH}_{3}\right)$, $1.12\left(1 \mathrm{H}, \mathrm{m},-\mathrm{CHOH}-\mathrm{CH} H_{a}\right), 1.54\left(1 \mathrm{H}, \mathrm{m},-\mathrm{CHOH}-\mathrm{CH} H_{b}\right), 1.76(\mathrm{H}, \mathrm{m},-\mathrm{CHMe}), 2.98(1 \mathrm{H}, \mathrm{s}$, exch. $\left.\mathrm{D}_{2} \mathrm{O},-\mathrm{OH}\right), 3.44\left(1 \mathrm{H}, \mathrm{d}, J 10.0,-\mathrm{SO}_{2} \mathrm{CHCH}=\mathrm{CH}\right), 4.62(1 \mathrm{H}, \mathrm{m},-\mathrm{CHOH}-), 5.01(1 \mathrm{H}, \mathrm{d}, J$ 17.3, $\left.-\mathrm{CH}=\mathrm{CH} H_{\text {trans }}\right), 5.38\left(1 \mathrm{H}, \mathrm{d}, J 10.3,-\mathrm{CH}=\mathrm{CH} H_{\text {cis }}\right), 6.03(1 \mathrm{H}, \mathrm{dt}, J 17.0,10.0$ and 10.0, $\left.\mathrm{CH}=\mathrm{CH}_{2}\right), 7.58(2 \mathrm{H}, \mathrm{m}, \mathrm{Ar} H), 7.65(1 \mathrm{H}, \mathrm{m}, \mathrm{Ar} H)$ and $7.84(2 \mathrm{H}, \mathrm{d}, J 8.0, o-\mathrm{Ar} H) \mathrm{ppm} ; \delta_{\mathrm{C}}(100$ $\mathrm{MHz}) 21.5\left(\mathrm{CH}_{3}\right), 22.5\left(\mathrm{CH}_{3}\right), 23.8(-\mathrm{CHMe}), 43.0\left(\mathrm{CH}_{2}\right), 65.8(-\mathrm{CHOH}-), 73.3\left(-\mathrm{CHSO}_{2}\right)$, $125.1\left(\mathrm{CH}=\mathrm{CH}_{2}\right), 125.5\left(\mathrm{CH}=\mathrm{CH}_{2}\right), 128.7$ (overlapping signals; $\left.C \mathrm{H}\right), 133.5(\mathrm{CH})$ and $137.0\left(C_{\mathrm{q}}\right)$ ppm.

3,4-syn-4-Hydroxy-6-methyl-3-phenylsulfonylhept-1-ene (28). Obtained as an oil, $v_{\max }(\mathrm{L})$ 3512,1305 and $1148 \mathrm{~cm}^{-1} ; \delta_{\mathrm{H}}(400 \mathrm{MHz}) 0.94\left(6 \mathrm{H}, \mathrm{d}, J 6.6,-\mathrm{CH}_{3}\right), 1.23(1 \mathrm{H}, \mathrm{m},-\mathrm{CHOH}-$ $\left.\mathrm{CH}_{a}\right), 1.37\left(1 \mathrm{H}, \mathrm{m},-\mathrm{CHOH}-\mathrm{CH} H_{b}\right), 1.96(\mathrm{H}, \mathrm{m},-\mathrm{CHMe}), 3.59(1 \mathrm{H}, \mathrm{dd}, J 10.1$ and 8.4, $\left.\mathrm{SO}_{2} \mathrm{CHCH}=\mathrm{CH}\right), 3.85\left(1 \mathrm{H}, \mathrm{s}\right.$, exch. $\left.\mathrm{D}_{2} \mathrm{O},-\mathrm{OH}\right), 4.41(1 \mathrm{H}, \mathrm{t}, J 10.0,8.2$ and 8.2, $-\mathrm{CHOH}-), 4.93$ $\left(1 \mathrm{H}, \mathrm{d}, J 17.0,-\mathrm{CH}=\mathrm{CH} H_{\text {trans }}\right), 5.26\left(1 \mathrm{H}, \mathrm{dd}, J 10.3\right.$ and $\left.1.0,-\mathrm{CH}=\mathrm{CH} H_{\text {cis }}\right), 5.55(1 \mathrm{H}, \mathrm{dt}, J 17.0$, 10.1 and 10.1, $\left.-\mathrm{CH}=\mathrm{CH}_{2}\right), 7.57(2 \mathrm{H}, \mathrm{m}, \mathrm{ArH}), 7.67(1 \mathrm{H}, \mathrm{m}, \mathrm{ArH})$ and $7.86(2 \mathrm{H}, \mathrm{d}, J$ 8.0, $o-\mathrm{Ar} H)$ ppm; $\delta_{\mathrm{C}}(100 \mathrm{MHz}) 20.7\left(\mathrm{CH}_{3}\right), 23.3\left(\mathrm{CH}_{3}\right), 23.5(-\mathrm{CHMe}), 43.3\left(\mathrm{CH}_{2}\right), 66.5(-\mathrm{CHOH}-), 75.9(-$ $\left.\mathrm{CHSO}_{2}\right), 123.7\left(\mathrm{CH}=\mathrm{CH}_{2}\right), 127.4\left(\mathrm{CH}=\mathrm{CH}_{2}\right), 128.4(\mathrm{CH}), 128.7(\mathrm{CH}), 133.5(\mathrm{CH})$ and 137.0 $\left(C_{\mathrm{q}}\right)$ ppm. HRMS $m / z$ 269.1203. Calc. for $\left[\mathrm{C}_{14} \mathrm{H}_{20} \mathrm{O}_{3} \mathrm{~S}+\mathrm{H}\right]^{+}: 269.1211$.

Protonolysis of the organozinc reagent derived from the bromosulfone 2. A solution of the bromosulfone $2(0.52 \mathrm{~g})$ in dry THF $(4 \mathrm{~mL})$ was added to zinc dust (5 equiv.), activated as described under Procedure B above. The mixture was refluxed during $2 \mathrm{~h}$ and then quenched with dilute sulfuric acid to yield an oil $(0.45 \mathrm{~g})$ that contained (NMR) a 50:50 mixture of the sulfone 2 and 3-phenylsulfonylprop-1-ene 29.

\section{Acknowledgements}

We thank Forbairt, the V. K. Krieble Fund and the Trinity Trust for financial support to ETG, and Dr John O'Brien for the NMR spectra. 


\section{References}

1. Gallagher, E. T.; Grayson, D. H. Org. Biomol. Chem. 2003, 1, 1374. http://dx.doi.org/10.1039/b300925b

2. Colonge, J.; Varagnat, J. Bull. Soc. Chim. Fr. 1961, 234.

3. Colonge, J.; Cayrel, S. P. Bull. Soc. Chim. Fr. 1965, 359.

4. Bellasoued, M.; Habbachi, F.; Gaudemar, M. Tetrahedron 1985, 41, 1299. http://dx.doi.org/10.1016/S0040-4020(01)96531-7

5. Rice, L. E.; Boston, M. C.; Finklea, H. O.; Suder, B. J.; Frazier, J. O.; Hudlicky, T. J. J. Org. Chem. 1984, 49, 1845. http://dx.doi.org/10.1021/jo00184a042

6. Hudlicky, T.; Natchus, M. G.; Kwart, L. D.; Colwell, B. L. J. Org. Chem. 1985, 50, 4300. http://dx.doi.org/10.1021/jo00222a020

7. Newman, M. S. J. Am. Chem. Soc. 1955, 77, 946. http://dx.doi.org/10.1021/ja01609a043

8. Pearson, D. E.; Cowan, D.; Beckler, J. D. J. Org. Chem. 1959, 24, 504. http://dx.doi.org/10.1021/jo01086a015

9. Eisch, J. J.; Galle, J. E. J. Org. Chem. 1979, 44, 3277. http://dx.doi.org/10.1021/jo01332a044

10. Culvenor, C. C. J.; Davies, W.; Savige, W. E. J. Chem. Soc. 1949, 2198. http://dx.doi.org/10.1039/jr9490002198 